Title:

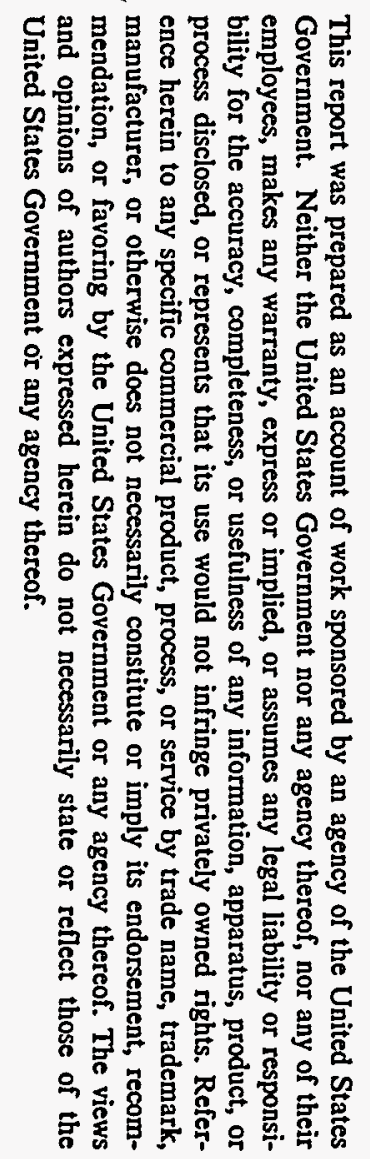

Submitted to:
Strongly Correlated Electronic Materials

Kevin Bedell, T-11

Robert Albers, $T=11$

Alexander Balatsky, T-11

Alan Bishop, T-11

Janez Bonca, T-11

James Gubernatis, T-11

Miklos Gulasci, T-11

Richard Silver, T-11

Stuart Trugman, T-11

\section{DOE Office of Scientific and Technical Information (OSTI)}

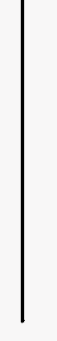

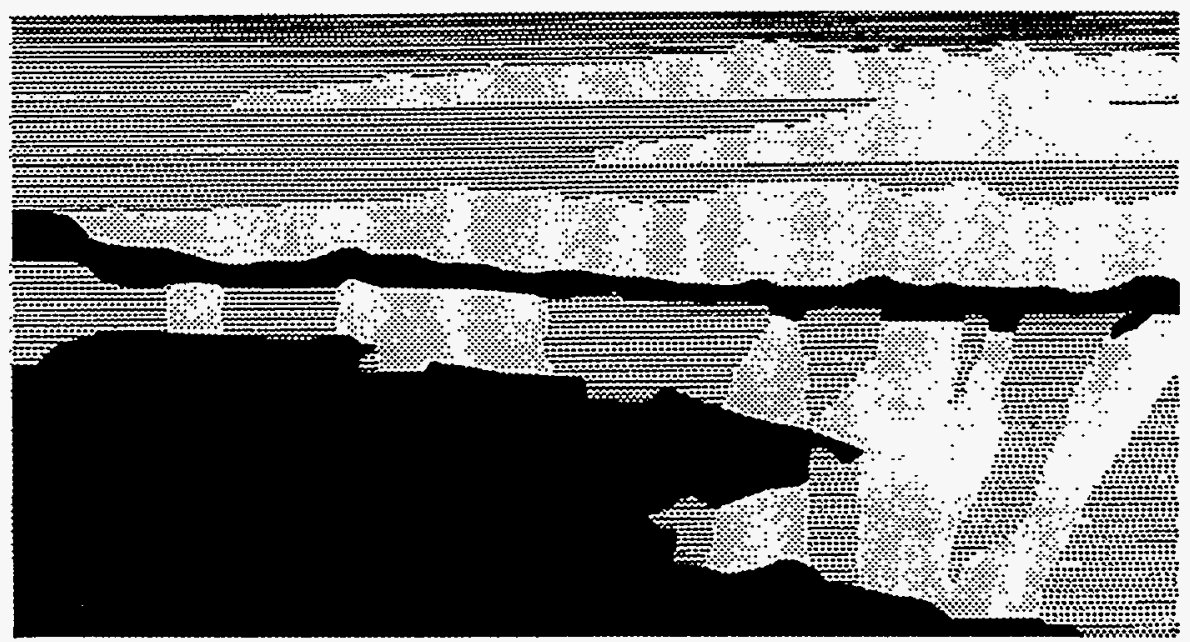

NATIONAL LABORATORY

Los Alamos National Laboratory, an affirmattve action/equal opportunity emoloyer, ts operated by the University of Calformia for the U.S. Department of Energy under contract W-7405-ENG-36. By acceptance this atti fo, the ppptisher recognizes that the U.S. Govemment retalns a nonexclusive, royalty-

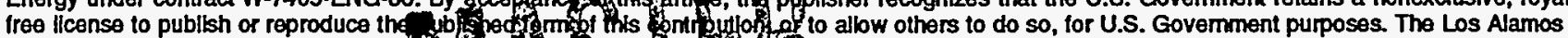

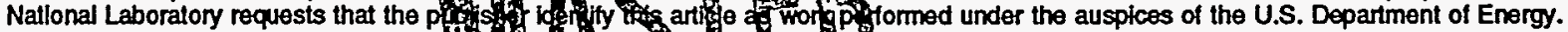




\title{
Strongly Correlated Electronic Materials
}

Kevin Bedell*, Robert Albers, Alexander Balatsky, Alan Bishop**, Janez Bonca, James Gubernatis, Miklos Gulasci, Richard Silver, and Stuart Trugman

\begin{abstract}
This is the final report of a three-year, Laboratory-Directed Research and Development (LDRD) project at the Los Alamos National Laboratory (LANL). Novel electronic materials characterized by strong electronic correlations display a number of unexpected, often extraordinary, properties. These are likely to play a major role in purpose-specific high-technology electronic materials of the future developed for electronic, magnetic and optical applications. This project sought to develop predictive control of the novel properties by formulating, solving and applying many-body models for the underlying microscopic physics. This predictive control required the development of new analytical and numerical many-body techniques and strategies for materials of varying strengths of interactions, dimensionality and geometry. The results of these techniques are then compared with experimental data on classes of novel materials, and the robust techniques are used to predict additional properties and motivate key additional experiments.
\end{abstract}

\section{Background and Research Objectives}

The last fifteen years has been a golden age for electronic materials with startling new discoveries of classes of phenomena and of complex electronic materials exhibiting them heavy fermions, electronic polymers, spin glasses, high-temperature superconductors (HTS), nanoscale materials, fullerenes, fractional quantum Hall materials, giant magneto-resistance materials, etc. These and related electronic materials are distinguished by a radical paradigm shift in solid state and materials science: away from traditional isotropic three-dimensional materials with simple unit cells, near-perfect crystal order, and linear, equilibrium responses; to anisotropic structures (clusters, chains and layers) with local, multiscale intrinsic and extrinsic

\footnotetext{
* Principal investigator

** LANL contact, e-mail: bishop_alan@lanl.gov
} 
disorder (texture) playing functional roles, and delicate balances of strong coupling between spin-charge-lattice degrees-of-freedom leading to a huge variety of broken-symmetry ground states (ferroelectric, martensitic, charge-density, spin-density, superconducting) driving strongly nonlinear and nonequilibrium excitations and responses. These novel electronic and structural states of matter and materials classes are, in the last few years, taking the first steps from academia to technology, since they offer the tunability (electronic, magnetic, optical) and breadth of characteristics demanded by high-technology requirements for next-generation "new materials." The need for control of advanced electronic materials now makes this theory and modeling guidance imperative for both fundamental understanding and technological competencies.

This project provided theory support for experimental activities in high-temperature superconductors, heavy-fermion materials with an emphasis on the Kondo insulators, and perovskite materials including the colossal magnetoresistance materials (CMR). In addition to these material-specific activities, we also addressed a number of issues that are common themes in these broad classes of materials. This included the study of these materials in magnetic fields, exploring properties like the field-induced metal-insulator transition, colossal magnetoresistance, spin-gap phenomena in HTS, the quantum Hall effect (QHE), and related issues. The properties of unconventional superconductors and impurity bound states in them were also studied. Another important theme is the metal-insulator transition and the nature of the metallic state in low-dimensional strongly correlated electronic materials.

\section{Importance to LANL's Science and Technology Base and National R\&D Needs}

Over the last ten years, Los Alamos has taken advantage of its interdisciplinary skills to establish a uniquely powerful presence in this arena of novel electronic materials. This excellence in fundamental research and interdisciplinary team-building now places LANL at the very forefront of technology innovation in electronic materials. The science developed on this project is at the forefront of new applications research. At the same time the materials studied present us with some of the most fundamental challenges in many-body theory. They inciude the colossal magneto-resistive materials that will have an enormous impact on the recording industry, heavy-fermions and, in particular, the Kondo insulators to be used for thermo-electric coolers, and the high temperature superconductors that are now on the brink of major applications with the recent breakthrough in HTS tape design. The impact of this project will be both on fundamental issues in correlated electron theory as well as on the characterization and design of new and novel electronic devices. This project supports Los Alamos core 
competencies in nuclear and advanced materials as well as theory, modeling and highperformance computing.

\section{Scientific Approach and Results to Date}

This project brought together a broad range of advanced many-body techniques to study key classes of strongly correlated electronic materials. The variety of physical phenomena exhibited necessitated a multi-technique approach. The exhibited techniques are complementary to one another and provide input and testing grounds for the various approaches. These include a number of analytic methods, field theoretic methods, bosonization, parquet, and weak coupling perturbation theory as well as variational techniques and mean field theories, $1 / \mathrm{N}$, inhomogeneous and unrestricted Hartree-Fock. There are also a variety of numerical methods, LDA, exact diagonalization, QMC, and MaxEnt. These techniques were applied to a broad spectrum of intellectually challenging and technologically important materials.

- We developed a microscopic understanding of the M-I transition. We studied the field induced M-I transition and its relation to CMR materials and the heavy-fermion insulators.

- We studied the optical properties of insulators and the dynamic correlation functions. Sum rules were used to study bounds on excitons in the heavy-fermion insulators as well as in semiconducting materials.

- In the metallic phase we studied extensions of the local Fermi liquid theory to include strong antiferromagnetic correlations. We explored the field induced metal-insulator transition in this model.

- We used numerical and analytical techniques to understand multi-band, PeierlsHubbard model Hamiltonians. Here the multi-band nature is critical to capturing essential hybridization effects, and the coupling of electronic and lattice degrees-offreedom.

- We studied doping states with respect to the stoichiometric ground states. Relevant polaron issues that we can now address include: ordering of polarons into superlattices and their melting as a function of temperature, quantum fluctuations, density, polaron size, and lattice commensurability.

- In the case of CMR materials recently studied at LANL (e.g., La1-x CaxMnO3) we developed a double-exchange Jahn-Teller model to study the systematic influence of temperature, magnetic field, and doping on magnetic, electronic and structural ground states, and on the structure and mobility of polarons as functions of mixed-valency. 
- We have solved essentially exactly for the quantum tunneling probability of a particle in the presence of inelastic degrees of freedom to calculate the effects of many-body electron-phonon interactions on the properties of realistic resonant tunneling diodes and quantum dots.

- We used the Eliashberg equations to investigate the complicated role of disorder in the strongly-coupled superconducting state. The weak localization theory and the Anderson localization problem have been developed as methods to address the role of impurities.

- An impurity in a d-wave superconductor generates a bound state, in analogy to the midgap impurity states in semiconductors. We have studied how these bound states produce significant contribution to the optical conductivity and, in principle, could account for the nontrivial temperature dependence of the conductivity.

- We have calculated the phase shifts and exponents entering into the transition probability rates for the $\mathrm{x}$-ray problem. We also calculated the decay rate of the electron into fractionally charged quasiparticles.

- We have begun for the first time to study systematically the ground-state properties of the Anderson lattice models. The results of this study are providing benchmarks of the fidelity of the model relative to experimental systems and of past approximations to the properties of the model.

- We have combined techniques with methods for the optimization of nuclear and phonon degrees of freedom, such as conjugate gradients, Monte Carlo and molecular dynamics. We are exploring a variety of possible real-world test cases such as the calculation of the atomic structure of amorphous and liquid silicon using semi-empirical tight-binding potentials.

\section{Publications}

[1] Silver, R. N.; Voter, A. F.; Kress, J. D., "Interatomic Potential for Covalent Materials from a Local Approximation to Tight Binding," Proceedings of Symposium on Computational Modeling of Materials and Processing at American Ceramics Meeting, May 1-3, 1995.

[2] Silver, R. N.; Roeder, H., "Densities of States of Mega-Dimensional Hamiltonian Matrices," International Journal of Modern Physics, 5, 735 (1994).

[3] Silver, R. N., "Ordering of Polarons in the Holstein t-J Model: An Application to La2x SrxNiO4+y," Europhysics Letters, 28, 257 (1994). 
[4] Silver, R. N.; Martz, H., "Applications of Quantum Entropy in Statistics," American Statistical Association, Toronto, published in 1994 Proceedings of the Statistical Computing Section, 61 (1994).

[5] Silver, R. N.; Voter, A. F.; Kress, J. D., Roeder, H., "Polynomial Approximations for Materials Simulations," published in Simulation Multiconference '95 Proceedings, High Performance Computing, ed. A. Tentner, 200 (1995).

[6] Wang, L.; Bishop, A. R., "Charge density wave of a multiband Fermi liquid on a graphitic nanotubule," Phys Rev B, 51, 7407 (1995).

[7] Wang, W-Z.; Bishop, A. R.; Lu, Yu, "Dynamic Jahn-Teller-induced infrared absorption in a charged C60 molecule," Phys. Rev B, R50, 5016 (1994).

[8] Salkola, M. I.; Mustre de Leon, J.; Bishop, A. R., "Correlation-function analysis of nonadiabatic and nonlinear systems: polaron tunneling," Phys Rev B, 51, 8878 (1995).

[9] Davids, P. S.; et al, "Collective level crossings on microtubules," Phys Rev B, 49, 5682 (1994).

[10] Bedell, K. S., et al. (eds.), "Lattice and Multiband electronic effects in hightemperature superconductors," in Strongly Correlated Electronic Materials, Addison Wesley (1994).

[11] Schmeltzer, D.; Bishop, A. R., "Bosonization in two dimensions - a space-time tunneling approach," Phys Rev B, 50, 12733 (1994).

[12] Spicci, M.; Salkola, M. I.; Bishop, A. R., "Coherence and dynamics of polarons in the presence of disorder," J. Phys. C., 6, 361 (1994).

[13] Salkola, M. I.; Bishop, A. R.; Trugman, S. A.; Mustre de Leon, J., "CorrelationFunction Analysis of Nonadiabatic and Nonlinear Systems: Polaron Tunneling," Phys Rev B, 51, 8878 (1995).

[14] Doniach, S.; Fu, Castor; Trugman, S. A., "Kondo Insulators: Are Simple Theories Good Enough?," Physica B, 199, 450 (1994).

[15] Migliori, A.; Sarrao, J. L.; Mandrus, D., Fisk, Z.; Balatsky, A.; Trugman, S. A., Thompson, J. D.; Maple, M. B., "Ultrasound Studies of U2Zn17 and UCu5," Physica B, 199, 36 (1994).

[16] Salkola, M. I.; Bishop, A. R.; Mustre de Leon, J.; Trugman, S. A., "Dynamic polaron Tunneling in $\mathrm{YBa} 2 \mathrm{Cu} 307$ : Optical Response and Inelastic neutron Scattering," Phys. Rev. B, 49, 3671 (1994).

[17] Inui, M.; Trugman, S. A.; Abrahams, E., "Unusual Properties of Mid-Band States in Systems With Off-Diagonal Disorder, Phys Rev B, 49, 3190 (1994). 
[18] Bonca, J.; Gubernatis, J. E., "Real-time dynamics from imaginary-time quantum Monte Carlo simulations: tests on oscillator chains," Phys. Rev B, 50, 10547 (1994).

[19] Balatsky, A. V.; Rosengren, A.; Altshuler, B. L., "Impurities and Quasi-One Dimensional Transport in a D-wave Superconductor," Phys Rev Lett, 73, 720 (1994).

[20] Bonca, J.; Balatsky, A. V., "Composite Operators for BCS Pairing," ZhETP Letters, 59, 202 (1994).

[21] Balatskyu, A. V.; Pines, D.; Monthous, P., "The Influence of Imperfections on the Low Temperature Properties of a D-wave Superconductors," Phys Rev B, 50, 582 (1994).

[22] Salkola, M. I.; Balatsky, A. V., "Superconducting Fluctuations in Multi-band 1D Hubbard Model," Phys Rev B, 51, 15267 (1995).

[23] Balatsky, A. V.; Salkola, M. I.; Rosengren, A., "Impurity Induced Bound States in DWave Superconductor," Phys Rev, 51, 15547 (1995).

[24] Balatsky, A. V., Abrahams, E., "Odd Time Magnetic Correlations and Chiral Spin Nematic," Phys Rev Lett, 74, 1004 (1995).

[25] Abrahams, E.; Balatsky, A. V.; Scalapino, D. J.; Schrieffer, J. R., "Properties of Odd Gap Superconductors, Phys Rev B, 52, 1271 (1995).

[26] Balatsky, A. V., Matveenko, S., "Dynamical Properties of Quantum hall States," Phys Rev, 52, 12 (1995).

[27] Bedell, K. S.; Farinas, P. F., "Kohn's Theorem and Correlation Functions for a Fermi Liquid," Phys Rev. Lett, 74, 4285 (1995).

[28] Engelbrecht, J.; Bedell, K. S., "Robustness of a Local Fermi Liquid Against Ferromagnetism and Phase Separation," Phys. Rev. Lett, 74, 4265 (1995).

[29] Sanchez-Castro, C.; Cooper, B.; Bedell, K. S., "Consequences of Competing Hybridization for Magnetic Ordering in Correlated-Electron Lattices," J. Appl. Phys., 76, 6130 (1994).

[30] Gulacsi, M.; Bedell, K. S., "Theory of the Metal-Insulator Transition in Strongly Correlated Electron Systems," Integrated Ferroelectrics, 6, 265 (1995).

[31] Gulacsi, M.; Bedell, K. S., "Spectral Function of the 1D Hubbard Model," Physica B, 199, 492 (1994).

[32] Bedell, K. S., "Lectures on Fermi Liquid Theory," The Canberra Summer School on Modern Perspectives in Many-Body Physics, World Scientific, 119 (1994). 